\title{
Características cinemáticas de potros da raça Brasileiro de Hipismo nos sucessos e insucessos dos saltos em liberdade
}

\author{
Godoi, F.N. ${ }^{1}$; Bergmann, J.A.G. ${ }^{2}$; Almeida, F.Q. ${ }^{3}$; Miranda, A.L.S. ${ }^{2}$; Santos, D.C.C. ${ }^{2}$; Siqueira, A.C.B. ${ }^{2}$; Pereira, M.B. ${ }^{3}$ e \\ Andrade, A.M. ${ }^{3}$
}

\begin{abstract}
'Departamento de Zootecnia. Universidade Federal da Bahia. Salvador-BA. Brasil.
${ }^{2}$ Escola de Veterinária. Universidade Federal de Minas Gerais. Belo Horizonte-MG. Brasil.

${ }^{3}$ Universidade Federal Rural do Rio de Janeiro. Seropédica-RJ. Brasil.
\end{abstract}

\section{PaLAVRAS CHAVE ADICIONAIS}

Biomecânica.

Equinos.

Esportes equestres.

\section{ADDITIONAL KEYWORDS}

Biomechanics.

Equine.

Show jumping.

INFORMACIÓN

Cronología del artículo.

Recibido/Received: 5.3.2015

Aceptado/Accepted: 15.6.2015

On-line: 16.9 .2015

Correspondencia a los autores/Contact e-mail:

fernandagodoi@gmail.com

\section{RESUMO}

Objetivou-se avaliar as características cinemáticas de potros nos saltos em liberdade, com sucesso ou insucesso. Foram avaliados 108 potros Brasileiro de Hipismo em cinco saltos, em liberdade, no obstáculo vertical com altura de 0,60 m aos 22-25 meses de idade; 0,80 m aos 29-32 meses de idade e 1,05 m aos 36-39 meses de idade. Foram filmados e avaliados 1.348 saltos, sendo 1.067 saltos com sucesso $(79,1 \%)$ e 281 saltos com insucesso $(20,9 \%)(p<0,01)$. Nos saltos com insucesso, $15,3 \%$ foram por derrube de obstáculo, $4,5 \%$ por desvio e 1,1\% por refugo. Os saltos com sucesso apresentaram maior distância da batida, de $1,17 \mathrm{~m} ; 1,51 \mathrm{~m}$ e 1,52 m na primeira, segunda e terceira avaliação, respectivamente. Observou-se que a velocidade do salto reduziu nos saltos com sucesso quando comparados com os saltos com insucesso, de $0,59 \mathrm{~m} / \mathrm{s}$ na primeira; de $0,19 \mathrm{~m} / \mathrm{s}$ na segunda e de $0,35 \mathrm{~m} / \mathrm{s}$ na terceira avaliação. A altura na cernelha durante o salto foi maior nos saltos com sucesso em relação aos com insucesso, sendo influenciada pela altura do obstáculo. Os ângulos da cabeça e do pescoço foram similares entre os saltos com sucesso e insucesso, exceto o ângulo da cabeça na primeira avaliação, de $48,6^{\circ}$ e $60,4^{\circ}$, nos saltos com sucesso e com insucesso, respectivamente. $O$ sucesso dos potros no salto foi de aproximadamente $80 \%$, com maior percentual de penalidades nos potros de 36-39 meses de idade, com obstáculo na altura de $1,05 \mathrm{~m}$. O derrube de obstáculos foi a principal penalidade durante a avaliação. As variáveis mais importantes para o sucesso do salto dos potros, independente do período de avaliação, foram a maior distância da batida e a menor velocidade do salto.

\section{Kinematics traits of young Brazilian Sport Horses in success or unsuccessful free jumping}

\section{SUMMARY}

This study was carried out to evaluate kinematics traits of young horses during free jumping regarding their success or not. One hundred and eight young Brazilian Sport Horses in five free jumpings attempts were record over a vertical obstacle of $0.60 \mathrm{~m}, 0.80$ and $1.05 \mathrm{~m}$ height at 22-25 to 29-32 and 36-39 months of age, respectively. A total of 1.348 jumping attempts were recorded and evaluated. From these, 1.067 jumping were successful (79.1\%) and 281 were failure $(20.9 \%)(p<0.01)$. Regarding failure jumpings, $15.3 \%$ were for knockdown, $4.5 \%$ by run-out and $1.1 \%$ by refusal. Successful jumpings presented larger take-off distance, of $1.17 \mathrm{~m}, 1.51 \mathrm{~m}$ and $1.52 \mathrm{~m}$ on the first, second and third evaluation, respectively. It was observed that jumping velocity decreased in successful jumpings when compared to unsuccessful ones, of $0.59 \mathrm{~m} / \mathrm{s}$ in the first; of $0.19 \mathrm{~m} / \mathrm{s}$ in the second and $0.35 \mathrm{~m} / \mathrm{s}$ in the third assessment. Height of withers during jumping was higher on successful jumps when compared to failures jumpings, and it was influenced by obstacle height. Angles of head and neck were similar between successful and unsuccessful jumpings, except the head angle at the first evaluation, of $48.6^{\circ}$ and $60.4^{\circ}$, in successful and failure jumps, respectively. Jumping success was approximately $80 \%$ in all evaluation, with the highest percentage of penalties regarding young horse of 36-39 months of age, with obstacle height of $1.05 \mathrm{~m}$. Knockdown was the main penalty throughout the evaluation periods. The most important variables for young horses successful jumping, regardless of the evaluation period, were higher take-off distance and lower jumping velocity.

\section{INTRODUÇÃO}

A avaliação visual é utilizada para julgar o movimento e desempenho dos equinos nas várias modalidades, assim como para seleção de animais para a reprodução e no diagnóstico de claudicações, porém, esse tipo de avaliação acarreta os riscos inerentes à subjetividade. A World Breeding for Sport Horses, entidade internacional que reúne as associações de raças de equinos utilizados na prática de esportes, da qual a raça Brasileiro de Hipismo é integrante, realiza testes para a aprovação de garanhões das raças e, um dos 
critérios de aprovação é o salto sobre obstáculo, porém os critérios de avaliação dos equinos durante o salto ainda são subjetivos. Dessa forma, a análise da cinemática deve ser utilizada para quantificar objetivamente os movimentos dos equinos, aumentando a precisão e propiciando melhor seleção de equinos para as modalidades hípicas (Clayton e Schamhardt, 2013).

Na prova de salto ocorrem muitas variáveis que condicionam o sucesso ou insucesso do conjunto cavalo-cavaleiro em ultrapassar os obstáculos, podendose citar o tempo de treinamento e a experiência do conjunto, as condições da pista, o tipo de obstáculo e, ainda, fatores ambientais como clima, público, sons, luminosidade, dentre outros. Porém, há fatores que são intrínsecos aos equinos e torna-os bons saltadores por si só, como a conformação corporal e a aptidão para o salto. Vários autores citam a importância de avaliar os equinos quando jovens em função da alta correlação das características cinemáticas de potros e de adultos no salto de obstáculos e, também, da alta correlação do salto em liberdade e salto montado (Santamaría et al., 2002; Wallin et al., 2003; Santamaría et al., 2004; Ducro et al., 2007). Dessa forma, esse trabalho teve como objetivo avaliar as características cinemáticas de potros nos saltos em liberdade, com sucesso ou insucesso.

\section{MATERIAL E MÉTODOS}

Foram utilizados 108 potros da raça Brasileiro de Hipismo, avaliados em três períodos durante o crescimento, de ambos os sexos, nascidos na mesma estação de reprodução na Coudelaria de Rincão - Exército Brasileiro, São Borja, RS.

No primeiro período de avaliação os potros apresentavam $22-25$ meses de idade, $412 \pm 39 \mathrm{~kg}$ de peso

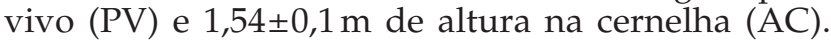
$\mathrm{Na}$ segunda avaliação os potros apresentavam 29-32 meses de idade, $461 \pm 40 \mathrm{~kg}$ PV e 1,62 $\pm 0,1 \mathrm{~m} \mathrm{AC} \mathrm{e,} \mathrm{na}$ terceira avaliação os potros apresentavam 36-39 meses de idade, $461 \pm 33 \mathrm{~kg}$ PV e 1,62 $\pm 0,1 \mathrm{~m} \mathrm{AC}$. Os potros foram domados aos 30-34 meses de idade, mas não foram montados e não tiveram treinamento de salto e de condicionamento físico. A doma foi conduzida em redondel utilizando cabresto com guia, nos andamentos passo, trote e galope.

$\mathrm{Na}$ avaliação cinemática, os potros foram conduzidos individualmente ao picadeiro coberto com dimensão de $45 \times 19$ metros, com uma face lateral preparada com fundo negro para a realização das filmagens, objetivando o controle da luminosidade exterior. Foram instalados dez pontos de luz próximos ao local de gravação visando melhorar a reflexibilidade dos marcadores. Dentro do picadeiro foi estruturado um coliseu, caracterizado como um corredor elíptico lateral ao picadeiro, possibilitando a condução dos equinos pelo centro. Imediatamente antes das filmagens, cada potro foi aquecido durante 10 minutos nos andamentos ao passo, ao trote e ao galope.

A linha de salto foi estruturada e constituída por um obstáculo principal em vertical precedido por um obstáculo de referência em forma de $X$ e com $0,45 \mathrm{~m}$ de altura, posicionado a 6,5 m do obstáculo principal.
O obstáculo vertical é um tipo de obstáculo móvel no qual o equino necessita transpor somente a altura. $\mathrm{O}$ obstáculo referência foi utilizado por vários autores (Powers e Harrison, 2000; Bobbert et al., 2005; Santamaría et al., 2006; Lewczuk, 2008) e visa garantir maior segurança aos animais por obrigar os potros a percorrer uma distância padronizada e abordarem o obstáculo vertical com maior facilidade.

O obstáculo vertical foi estruturado nas alturas de 0,60 m (22-25 meses de idade); 0,80 m (29-32 meses de idade) e 1,05 m (36-39 meses de idade). No intuito de melhor adaptação dos potros ao obstáculo, foram realizados dois saltos em alturas menores, com o obstáculo em forma de X. Em seguida, modificou-se o obstáculo principal para a forma de vertical e cada potro realizou, ao galope, cinco saltos em liberdade, consecutivos, em cada avaliação. Os saltos foram classificados em: 1) salto com sucesso - quando o potro executou o salto sobre o obstáculo vertical sem cometer nenhum tipo de penalidade; 2) salto com insucesso: quando houve algum tipo de penalidade no obstáculo vertical, sendo essas definidas como: derrube: quando o obstáculo caiu durante o salto devido ao toque com alguma parte do corpo do equino; desvio - quando o equino não realizou o salto, desviando-se lateralmente; e refugo - quando o equino parou na frente do obstáculo, não ultrapassando-o.

As imagens foram obtidas utilizando uma câmera Basler $A 602 f_{C}{ }^{\circledR}$ na forma bidimensional e com aquisição de imagens em $100 \mathrm{~Hz}$, a 16,3 metros do centro do obstáculo vertical. O aplicativo Simi Reality Motion Systems ${ }^{\circledR} 4.7$ foi utilizado para acionar a câmera e visualizar, armazenar e processar as imagens.

Para a avaliação cinemática dos potros durante o salto com sucesso e com insucesso (derrube de obstáculo) foram utilizados marcadores reflexivos circulares, com cinco centímetros de diâmetro, fixados em 18 pontos anatômicos da face corporal esquerda dos animais utilizados como referências (Lewczuk et al., 2006; Clayton e Schamhardt, 2013; Godoi et al., 2013) (figura 1). As variáveis mensuradas no corpo do equino e em relação ao obstáculo foram denominadas características de desempenho no salto, sendo estas: amplitude da passada anterior ao salto - comprimento da passada completa do membro torácico esquerdo anterior ao salto, utilizando o marcador 8 como referência; velocidade da passada anterior ao salto - velocidade horizontal calculada como a distância da amplitude da passada anterior ao obstáculo em relação ao tempo; amplitude do salto comprimento do salto, mensurado pela distância entre o contato do casco do membro pélvico esquerdo com o solo na batida (decolagem) até o contato deste mesmo casco com o solo na recepção (aterrissagem) utilizando o marcador 16 como referência; velocidade do salto velocidade horizontal calculada como a distância da amplitude do salto em relação ao tempo; distância da batida (decolagem) - distância entre o obstáculo e o casco do membro pélvico mais próximo ao obstáculo, imediatamente antes da fase de vôo, utilizando o marcador 16 ou 18 como referência; distância da recepção (aterrissagem) - distância entre o obstáculo e o casco torácico que toca primeiro o solo imediatamente após a fase de vôo, utilizando o marcador 8 ou 10 como referência; altura 


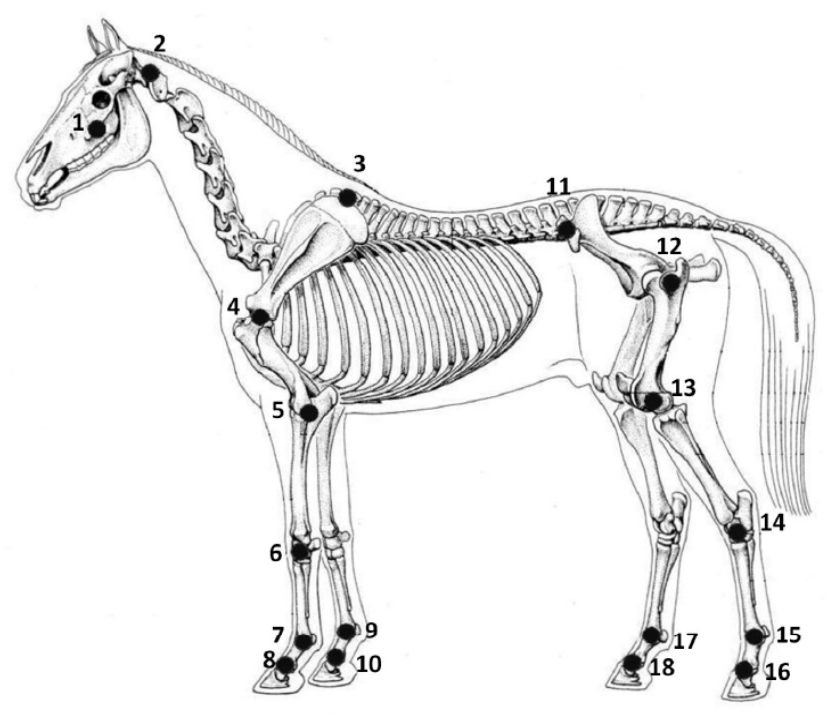

Figura 1. Posicionamento dos marcadores reflexivos: 1) ponto médio da crista facial; 2) porção cranial da face lateral da asa do atlas; 3) porção dorsal na cartilagem da escápula seguindo a linha da espinha da escápula; 4) área central da articulação escápulo-umeral, na região da cavidade glenóide da escápula e cabeça do úmero; 5) área central da articulação úmero-radial, na região do côndilo do úmero, fóvea capitular do rádio e incisura troclear da ulna; 6) terço médio lateral da articulação cárpica, região lateral do osso carpiano ulnar; 7) terço médio da face lateral da articulação metacarpofalângica do membro torácico esquerdo; 8) face lateral da articulação interfalângica proximal do membro torácico esquerdo; 9) terço médio da face medial da articulação metacarpofalângica do membro torácico direito; 10) face medial da articulação interfalângica proximal dos membros torácico direito; 11) ponto médio ventral da face lateral da tuberosidade coxal; 12) região média do trocanter maior do fêmur, na articulação coxo-femoral; 13) ponto lateral da articulação fểmoro-tibial; 14) terço médio lateral da articulação társica, na região lateral entre a base do calcâneo e osso tálo; 15) terço médio da face lateral da articulação metatarsofalângica do membro pélvico esquerdo; 16) face lateral da articulação interfalângica proximal do membro pélvico esquerdo; 17) terço médio da face medial da articulação metatarsofalẩngica do membro pélvico direito e 18) face medial da articulação interfalângica proximal do membro pélvico direito; de acordo com Schaller (1999) (Positions of the reflexive markers: 1) middle point of facial crest; 2) cranial portion of face lateral of the atlas wing; 3 ) dorsal portion of scapular cartilage following the line of the scapular spine; 4) central area of scapulohumeral joint, in the region of the glenoid cavity of the scapula and the head of the humerus; 5) central area of humeroradial joint in the region of the condyle of the humerus, the radial fossa, and the trochlear (semilunar) notch; 6) lateral middle third of the carpal joint, in the lateral region of the ulnar carpal bone; 7) middle third of the lateral face of the metacarpophalangeal joint of the left thoracic limb; 8) lateral face of the proximal interphalangeal joints of the left thoracic limb; 9) middle third of the medial face of the metacarpophalangeal joint of the right thoracic limb; 10) medial face of the proximal interphalangeal joints of the right forelimb; 11) ventral middle point of the lateral face of the coxal tuber; 12) middle region of the greater trochanter of the femur in the coxofemoral joint; 13) lateral point of the femorotibial joint; 14) lateral middle third of the tarsal joint, in the lateral region between the base of the calcaneus and the talus; 15) middle third of the lateral face of the metatarsophalangeal joint of the left pelvic limb; 16) lateral face of the proximal interphalangeal joints of the left pelvic limb; 17) middle third of the medial face of the metacarpophalangeal joint of the right pelvic limb, and 18) medial face of the proximal interphalangeal joints of the right pelvic limb; according Schaller (1999)). dos membros torácicos no salto - altura mínima dos membros torácicos em relação ao obstáculo, mensurado utilizando o marcador 7 ou a pinça como referência, no momento da passagem desse sobre o obstáculo; altura dos membros pélvicos no salto - altura dos membros pélvicos em relação ao obstáculo, utilizando o marcador 15 como referência, no momento da passagem desse sobre o obstáculo; altura da cernelha no salto - altura vertical máxima do equino durante a trajetória do salto até o solo, utilizando o marcador 3 como referência; deslocamento da cernelha no salto - distância horizontal utilizando o marcador 3 como referência, no momento da altura máxima da cernelha, que pode ser anterior ou posterior ao obstáculo, sendo padronizado em valor negativo e positivo, respectivamente.

As seguintes variáveis foram mensuradas exclusivamente no corpo dos equinos no salto de obstáculo e permitem qualificar o salto e foram denominadas características morfológicas dos equinos no salto. Em todas as variáveis do segmento torácico e pélvico que foram mensuradas respectivamente no tempo no qual o membro torácico esquerdo (marcador 7) e membro pélvico esquerdo (marcador 15), estavam sobre o obstáculo, sendo ângulo da cabeça - ângulo ventral formado pela interseção das linhas entre os marcadores 1-2 e 2-3; ângulo do pescoço - ângulo ventral formado pela interseção das linhas entre os marcadores 2-3 e 3-4; ângulo escápulo-umeral - ângulo caudal formado pela interseção das linhas entre os marcadores 3-4 e 4-5; ângulo úmero-radial - ângulo cranial formado pela interseção das linhas entre os marcadores 4-5 e 5-6; ângulo rádio-metacárpico - ângulo caudal formado pela interseção das linhas entre os marcadores 5-6 e 6-7; ângulo coxo-femoral - ângulo cranial formado pela interseção das linhas entre os marcadores 11-12 e 12-13; ângulo fêmoro-tibial - ângulo caudal formado pela interseção das linhas entre os marcadores 12-13 e 13-14; ângulo tíbio-metatarsico - ângulo cranial formado pela interseção da linha entre os marcadores 13-14 e 14-15; ângulo cernelha-boleto - ângulo cranial formado pela interseção da linha entre os marcadores 3-11 e 11-15; distância vertical entre as articulações escápulo-umeral e metacarpofalângica - distância vertical ou a diferença entre dois pontos compreendida entre os marcadores 4-7; distância vertical entre as articulações úmero-radial e metacarpofalângica - distância vertical ou a diferença entre dois pontos compreendida entre os marcadores 5-7; distância linear entre as articulações úmero-radial e metacarpofalângica - distância compreendida entre os marcadores 5-7 e distância vertical entre as articulações fêmoro-tibial e metatarsofalângica - distância vertical ou a diferença entre dois pontos compreendida entre os marcadores 13-15. Essa última mensuração pode ser negativa se a articulação metatarsofalângica estiver acima da altura da articulação fêmoro-tibial. Essas variáveis foram adaptadas de Bobbert et al. (2005); Santamaría et al. (2006); Lewczuck et al. (2006); Lewczuck e Ducro (2012).

Os resultados foram submetidos à análise estatística descritiva com os valores médios, mínimos e máximos e coeficiente de variação. Os resultados percentuais dos saltos com sucesso e insucesso e os percentuais das penalidades dentro dos saltos com insucesso foram 
Tabela I. Quantificação dos saltos dos cavalos jovens com sucesso e insucesso e classificação dos saltos com insucesso nas três avaliações (Quantification the jumping of young horses with success and failure and classification of failure jumps).

\begin{tabular}{|c|c|c|c|c|c|c|c|c|}
\hline \multirow{3}{*}{ Itens } & \multicolumn{8}{|c|}{ Período de avaliação (Idade) } \\
\hline & \multicolumn{2}{|c|}{ 22-25 meses } & \multicolumn{2}{|c|}{ 29-32 meses } & \multicolumn{2}{|c|}{ 36-39 meses } & \multicolumn{2}{|c|}{ Total } \\
\hline & $\mathrm{n}$ & $\%$ & $\mathrm{n}$ & $\%$ & $\mathrm{n}$ & $\%$ & $\mathrm{n}$ & $\%$ \\
\hline \multicolumn{9}{|c|}{ CLASSIFICAÇÃO DOS SALTOS ${ }^{*}$} \\
\hline Sucesso & 446 & $84,9^{A}$ & 352 & $83,2^{\mathrm{A}}$ & 269 & $67,3^{A}$ & 1.067 & 79,1 \\
\hline Insucesso & 79 & $15,1^{\mathrm{B}}$ & 71 & $16,8^{\mathrm{B}}$ & 131 & $32,7^{\mathrm{B}}$ & 281 & 20,9 \\
\hline Total & 525 & 100 & 423 & 100 & 400 & 100 & 1.348 & 100 \\
\hline \multicolumn{9}{|c|}{ SALTOS COM INSUCESSO* } \\
\hline Derrubes & 51 & 9,7 & 50 & 11,8 & 105 & 26,2 & 206 & 15,3 \\
\hline Desvios & 26 & 5,0 & 15 & 3,6 & 20 & 5,0 & 61 & 4,5 \\
\hline Refugos & 02 & 0,4 & 06 & 1,4 & 06 & 1,5 & 14 & 1,1 \\
\hline \multicolumn{9}{|c|}{ SALTOS COM INSUCESSO: DERRUBES COM OS MEMBROS ${ }^{*}$} \\
\hline Torácico & 24 & $48^{A}$ & 23 & $45^{\mathrm{A}}$ & 74 & $70^{A}$ & 121 & 59 \\
\hline Pélvico & 26 & $52^{A}$ & 28 & $55^{A}$ & 31 & $30^{B}$ & 85 & 41 \\
\hline Total & 51 & 100 & 50 & 100 & 105 & 100 & 206 & 100 \\
\hline
\end{tabular}

*Letras diferentes na coluna diferem pelo teste de Qui-quadrado $(p<0,01)$.

comparados pelo teste de Qui-quadrado $(\mathrm{p}<0,01)$ utilizando o Sistema de Análises Estatísticas e Genéticas.

\section{RESULTADOS E DISCUSSÃO}

Foram analisados 1.348 saltos, sendo $79,1 \%$ com sucesso e 20,9\% com insucesso, diferindo significativamente $(\mathrm{p}<0,01)$. O maior percentual de penalidades foi o derrube de obstáculo, com 15,3\% do total de saltos e considerando apenas os saltos com insucessos, o percentual foi de $73,3 \%(p<0,01)$. Os potros derrubaram os obstáculos com maior frequência com os membros torácicos (59\%) em relação aos membros pélvicos (41\%) $(\mathrm{p}<0,01)$. O percentual de desvio do obstáculo foi de $4,5 \%$ do total de saltos e, considerando apenas os saltos com insucessos, o percentual foi de $21,7 \%$. Houve $1,1 \%$ de refugo do obstáculo, do total de saltos e de $4,9 \%$ considerando apenas os saltos com insucesso (tabela I).

Houve efeito significativo na porcentagem de saltos com sucesso em relação ao salto com insucesso em todos os períodos de avaliação, sendo os saltos com sucesso foram mais frequentes. Porém, numericamente, houver maior porcentagem de saltos com insucesso no terceiro período de avaliação, de 32,7\%. Dentro dos saltos com insucesso, houve maior porcentagem de derrubes em todos os períodos de avaliação, seguindo do desvio e de refugos. Porém, numericamente, houve maior frequência de derrubes no terceiro período de avaliação, quando o salto é maior e os equinos já haviam repetidos os protocolos duas vezes. Não é aconselhável realizar análise estatística para verificar diferenças entre os períodos de avaliação, pois há o efeito do crescimento dos equinos, ou seja, o efeito de ambiente é muito diferente.

Dos saltos com derrube na terceira avaliação, 70\% foram causados com os membros torácicos. De forma similar, Santamaría et al. (2002), avaliando o salto com sucesso de potros com seis meses de idade sobre o obstáculo de $0,60 \mathrm{~m}$, observaram que os potros flexionam significativamente mais os membros pélvicos quando comparados aos membros torácicos. Com esses resultados, sugere-se que a altura do obstáculo vertical, de 1,05 metros, proporcionou maior dificuldade para ser ultrapassado pelos potros com 36-39 meses de idade e, pode-se inferir que obstáculo com menor altura nessa idade não consistiria em desafio para os animais com aptidão para o salto.

As variáveis cinemáticas amplitude e velocidade da passada anterior ao salto e distância da batida são variáveis de preparação para o salto, que serão alteradas de acordo com o tipo e altura do obstáculo. Houve aumento dessas características em $0,21 \mathrm{~m} ; 0,32 \mathrm{~m} / \mathrm{s}$ e $0,35 \mathrm{~m}$, respectivamente, no obstáculo com $0,60 \mathrm{~m}$ de altura para o obstáculo com 1,05 m nos saltos com sucesso (tabela II). Essas características possibilitam aos equinos ajustar a distância para a decolagem e a velocidade adequada para ultrapassar o obstáculo, que também estão associadas a insucessos nos saltos.

Apesar da presença do obstáculo de referência, os potros ajustaram a amplitude e a velocidade da passada anterior ao salto, influenciando a distância da batida e, consequentemente, as características do salto. Nas avaliações, observou-se que nos saltos com sucesso e insucesso houve um padrão de aumento da amplitude e velocidade da passada anterior ao salto e redução na distância da batida, sendo a maior diferença observada entre os saltos com sucesso e insucesso na primeira avaliação, quando compara-se o saltos de sucesso com os de insucesso.

Em saltos montados, ou seja, com a presença do cavaleiro, a amplitude e velocidade da passada anterior ao obstáculo e distância da batida podem ser ajustadas de acordo com a experiência do cavaleiro e a sugestão do treinador. Santamaría et al. (2002) avaliando saltos com sucesso de potros com seis meses de idade, em liberdade, com obstáculo referência a seis metros do obstáculo vertical de 0,60 m, observaram amplitude anterior ao salto variando de 2,0-2,5 m. 


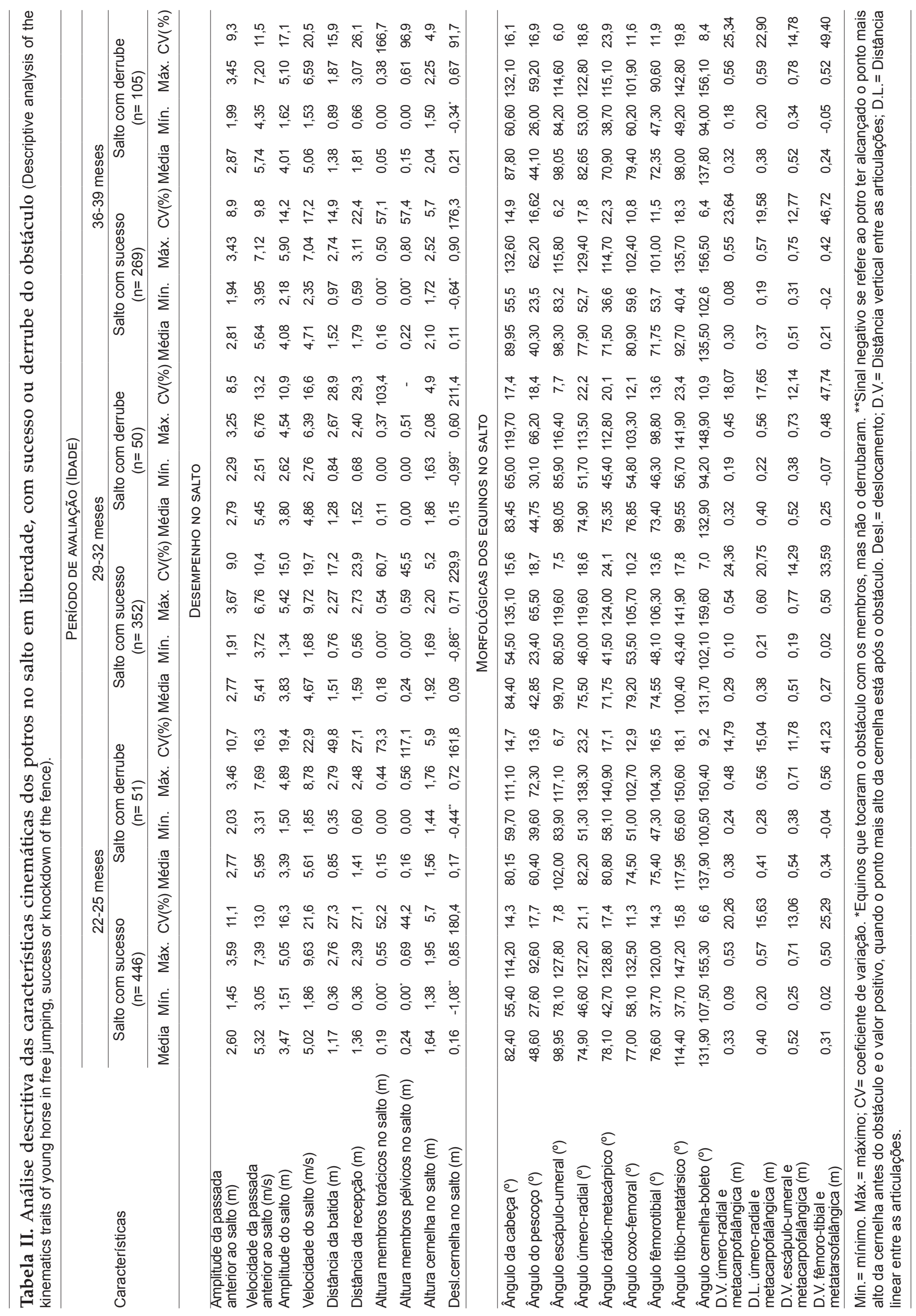


No presente trabalho, os potros ampliaram a saída para o salto, ou seja, apresentaram maior valor de distância da batida, nos saltos com sucesso em relação aos saltos com insucesso, de 0,32 m; 0,23 m e 0,14 m de diferença na primeira, segunda e terceira avaliação, respectivamente. Observou-se também, aumento dessa variável com o subsequente aumento da altura do obstáculo.

A amplitude e a velocidade do salto e a distância da recepção também são influenciadas pelo tipo e altura do obstáculo. Essas características podem ser consideradas consequências das citadas anteriormente, pois a maior ou menor distância da batida irá, consequentemente, influenciar nestes valores, especialmente no obstáculo vertical que não possui largura para ser ultrapassado. Observou-se que a amplitude do salto aumentou em função da altura do obstáculo, em 0,36 m e $0,25 \mathrm{~m}$, nos obstáculos com $0,60 \mathrm{~m}$ para $0,80 \mathrm{~m}$ e de 0,80 para $1,05 \mathrm{~m}$, respectivamente. Também foi observado que a velocidade do salto apresentou redução nos saltos com sucesso em relação aos saltos com insucesso, de $0,59 \mathrm{~m} / \mathrm{s}$ na primeira avaliação; de $0,19 \mathrm{~m} / \mathrm{s}$ na segunda avaliação e de $0,35 \mathrm{~m} / \mathrm{s}$ na terceira avaliação. A distância da recepção e a amplitude do salto são variáveis que caracterizam a aterrissagem do equino, com os membros torácicos e pélvicos, respectivamente, e o início de um novo galope, caracterizando o final do salto. A aterrissagem é importante fase do salto especialmente durante as provas hípicas, nas quais os obstáculos são sequenciais. A distância da recepção foi maior nos saltos com maior altura, com 0,43 m de diferença nos saltos com sucesso no obstáculo com $0,60 \mathrm{~m}$ e 1,05 m. Deuel e Park (1991) observaram que os melhores saltadores apresentaram menor velocidade do salto, menor distância da batida e menor distância da recepção durante competição de salto não caracterizando uma parábola, em obstáculo com 1,60 m de altura e 1,00 m de largura. Vale ressaltar que a velocidade é uma relação entre tempo e a distância mensurada entre dois pontos horizontais, não considerando a altura; dessa forma, a associação observada entre salto mais veloz a um salto com maior percentual de derrube, pode-se, também, relacionar a um salto mais rasteiro.

As características amplitude do salto, distância da batida, distância da recepção, altura da cernelha no salto e deslocamento da cernelha no salto possibilitam descrever a trajetória do salto realizada pelo potro e indica as distâncias horizontais, anterior e posterior, e distância vertical da parábola do salto em relação ao obstáculo.

Na literatura, é comumente observado que a parábola do salto é avaliada utilizando-se como referência o centro de gravidade (Powers e Harrison, 1999; Powers e Harrison, 2000; Santamaría et al., 2004; Santamaría et al., 2006). No entanto, devido às limitações técnicas, o centro de gravidade não foi estimado nesse trabalho, optando-se pelo ponto na cernelha por ser próximo ao centro de gravidade e por indicar a altura máxima alcançada pelo equino na fase do salto. Devido às observações práticas e experimentais pode-se sugerir que a altura máxima para ultrapassar o obstáculo é menos importante como causa do derrube do obstáculo em relação à altura dos membros sobre o obstáculo. Desse modo, é menos interessante para o sucesso do salto que os equinos projetem seu corpo muito acima da altura necessária para ultrapassar o obstáculo sem, contudo, apresentar adequada flexão dos membros.

Como esperado, a altura da cernelha no salto aumentou com a elevação da altura do obstáculo vertical, de $1,64 \mathrm{~m}$ no obstáculo à $0,60 \mathrm{~m}$ de altura, para 2,10 m no obstáculo com $1,05 \mathrm{~m}$, nos saltos com sucesso, ou seja, uma diferença de $46 \mathrm{~cm}$. A diferença na altura na cernelha dos potros em estação forçada nesse mesmo período foi de oito centímetros. Dessa forma pode-se inferir que o valor absoluto da diferença nas alturas dos saltos na primeira e terceira avaliação foi de $38 \mathrm{~cm}$. O valor médio dessa variável foi ligeiramente maior nos saltos com sucesso em relação aos saltos com insucesso.

Nos saltos com sucesso na segunda e terceira avaliações, a altura máxima com a qual o equino ultrapassou o obstáculo apresentou-se mais próxima do obstáculo, ou seja, obteve-se menores valores do deslocamento horizontal da cernelha. Schlup et al. (2014) observaram diferenças no deslocamento horizontal da cernelha nos saltos em equinos antes e após treinamento, com alteração da trajetória do salto em função do treinamento.

Os ângulos da cabeça e do pescoço estão diretamente relacionados ao movimento de báscula durante a trajetória do salto. A movimentação desses segmentos acompanha as fases do salto, proporcionando maior equilíbrio para o equino. A cabeça e o pescoço são responsáveis por aproximadamente $10 \%$ do peso total do equino adulto (Buchner et al., 1997) e é capaz de deslocar o centro de gravidade do cavalo durante a locomoção. Os valores dos ângulos da cabeça e do pescoço foram similares entre os saltos com sucesso e insucesso, exceto o ângulo da cabeça na primeira avaliação, de $48,6^{\circ}$ e $60,4^{\circ}$, nos saltos com sucesso e com insucesso, respectivamente.

O ângulo escápulo-umeral exerce influência na báscula do pescoço e no recolhimento dos membros torácicos, uma vez que as articulações e partes anatômicas envolvidas estão diretamente conectadas, possibilitando que o movimento de uma articulação influencie no da outra. Esse ângulo que é importante durante a aterrissagem para a absorção do impacto, apresentou valores médios variando de $98,1^{\circ}$ a $102,0^{\circ}$ durante toda a avaliação.

Os ângulos úmero-radial e rádio-metacárpico são responsáveis pelo recolhimento dos membros torácicos. Nos saltos com sucesso os valores médios desses ângulos foram, respectivamente, de $74,9^{\circ}$ e $78,1^{\circ}$ na primeira avaliação; de $75,5^{\circ}$ e $71,8^{\circ}$ na segunda avaliação; e de $77,9^{\circ}$ e $71,5^{\circ}$ na terceira avaliação. Observa-se que houve redução no valor médio do ângulo rádio-metacárpico da primeira avaliação para a terceira avaliação, de $6,6^{\circ}$, indicando maior flexão dos membros torácicos quando o obstáculo foi de $1,05 \mathrm{~m}$. Os equinos que apresentaram menores valores desses ângulos também apresentaram maiores valores da altura dos membros torácicos sobre o obstáculo o que implica em menor possibilidade de derrube do obstáculo (Santamaría et al., 2002; Bobbert et al., 2005). Powers e Harrison (2000) 
avaliando equinos de três a cinco anos de idade no salto em liberdade, verificaram que os equinos que se destacaram como melhores saltadores apresentaram maior flexão dos membros torácicos, ou seja, menores valores dos ângulos.

Em relação aos membros pélvicos é desejável que equinos com aptidão para o salto apresentem os maiores valores dos ângulos coxo-femoral e tíbio-metatársico para, durante o salto, proporcionar menor possibilidade de derrube do obstáculo (Bobbert et al., 2005; Santamaría et al., 2006). No presente trabalho, os potros apresentaram valores do ângulo fêmorotibial, na primeira, segunda e terceira avaliação, respectivamente, de $76,6^{\circ} ; 74,5^{\circ}$ e $71,8^{\circ}$ nos saltos com sucesso, indicando uma tendência para a redução desse ângulo com o aumento da altura do obstáculo. Santamaría et al. (2006) analisaram a biomecânica do salto em equinos aos seis meses, aos quatro anos e aos cinco anos de idade, verificando que os melhores saltadores apresentavam menor ângulo fêmorotibial.

O ângulo cernelha-boleto é a variável que faz a conexão do segmento torácico e pélvico e desse modo, relaciona o movimento da coluna e a altura na qual os membros pélvicos ultrapassam o obstáculo durante o salto, sendo desejado o maior valor desse ângulo. Em todo o estudo, o valor desse ângulo nos potros saltando com sucesso ou com insucesso variaram de $102,1^{\circ}$ a $159,6^{\circ}$ e de 94,0 a $156,1^{\circ}$, respectivamente. É interessante ressaltar que em estudo realizado por Van Weeren et al. (1992), foi observado que o deslocamento da pele pode influenciar os valores das variáveis mensuradas, com maior influência na região do tronco em relação às regiões distais, podendo essas ser até desconsideradas o deslocamento da pele. O ângulo cernelha-boleto é considerado pela literatura como um dos parâmetros mais importantes na avaliação dos equinos de salto. Bobbert et al. (2005) e Santamaría et al. (2006) verificaram diferenças no ângulo cernelha-boleto entre os melhores e os piores equinos de salto aos seis meses de idade, saltando obstáculo de $0,60 \mathrm{~m}$ de altura, e com equinos de cinco anos de idade, saltando obstáculo de $1,15 \mathrm{~m}$ de altura, sendo os melhores saltadores aqueles que apresentaram maior angulação. No presente trabalho, ocorreram saltos nos quais os obstáculos foram tocados pelos potros, mas não derrubados. Esses saltos foram contabilizados como sucesso e, talvez por esse fato, não foram observadas diferenças expressivas nas angulações supracitadas nos saltos com sucesso e insucesso.

Há importância em realizar trabalhos com resultados dos saltos com sucesso ou insucesso, visando um estudo completo e detalhado da biomecânica do salto dos equinos utilizando todo o universo de características. Observa-se, na literatura corrente, que os equinos são classificados em melhores e piores saltadores somente pela utilização dos resultados dos saltos com sucesso (Bobbert et al., 2005; Santamaría et al., 2006; Schlup et al., 2014) e sendo os saltos com insucesso descartados. Sendo assim, é interessante estipular uma quantidade de saltos para os equinos e verificar qual a reação desses animais perante o obstáculo: salto, refugo ou desvio. Esse protocolo pode ser aplicado especialmente em potros visando avaliar a aptidão para o salto.

\section{CONCLUSÃO}

O sucesso dos potros no salto sobre obstáculo nas diferentes idades foi de aproximadamente $80 \%$, com maior percentual de penalidades nos potros de 36 a 39 meses de idade, com o obstáculo na maior altura. O derrube de obstáculos foi a principal penalidade em todos os períodos de avaliação.

As variáveis mais importantes para o sucesso do salto dos potros, independente da idade nas avaliações, foram a maior distância da batida e a menor velocidade no salto.

\section{AGRADECIMENTOS}

À Coudelaria de Rincão - Exército Brasileiro, pela utilização dos animais. Conselho Nacional de Desenvolvimento Científico e Tecnológico (CNPq) e a Fundação de Amparo à Pesquisa de Minas Gerais (FAPEMIG), pelo suporte financeiro para o estudo.

\section{BIBLIOGRAFIA}

Bobbert, M.; Santamaría. S.; Van Weeren. P.R.; Back, W. and Barneveld, A. 2005. Can jumping capacity of adult show jumping horses be predicted on the basis of sub maximal free jumps at foal age? $A$ longitudinal study. Vet J, 170: 212-221.

Buchner, H.H.F.; Savelberg, H.H.CM.; Schamhardt, H.C. and Barneveld, A. 1997. Inertial properties of Dutch Warmblood horses. J Biomech, 30: 653-658.

Clayton, H.M. and Schamhardt, H.C. 2013. Measurement techniques for gait analysis. In: Back, W.; Clayton, H.M. Equine locomotion, $2^{a}$ ed. Ed. Saunders. London. p. 31-60.

Deuel, N.R. and Park, J. 1991. Kinematic analysis of jumping sequences of Olympic Show Jumping horses. Equine Exerc Phys, 3: 158-166.

Ducro, B.J.; Koenen, E.P.C.; Van Tartwijk, J.M.F.M. and Bovenhuis, H. 2007. Genetic relations of movement and free-jumping traits with dressage and show-jumping performance in competition of Dutch Warmblood horses. Livest Sci, 107: 227-234.

Godoi, F.N.; Bergmann, J.A.G.; Almeida, F.Q.; Santos, D.C.C.; Miranda, A.L.S.; Oliveira, F.O.; Oliveira, J.E.G.; Kaipper, R.R. e Andrade, A.M. 2013. Morfologia de potros da raça Brasileiro de Hipismo. Ciên Rur, 43: 736-742.

Lewczuk, D. 2008. Young horse response on changing distance in free jumping combination. Anim, 2: 1651-1657.

Lewczuk, D. and Ducro, B. 2012. Repeatability of free jumping parameters on tests of different duration. Livest Sci, 146: 22-28.

Lewczuk, D.; Sloniewski, Z. and Reklewski, Z. 2006. Repeatability of the horse's jumping parameters with and without the rider. Livest Sci, 99: 125-130.

Powers, P. and Harrison, A. 1999. Models for biomechanical analysis of jumping horses. J Equine Vet Sci, 19: 799-806.

Powers, P.N.R. and Harrison, A.J. 2000. A study on the techniques used by untrained horses during loose jumping. J Equine Vet Sci, 20: 844-850.

Santamaría, S.; Back, W.; van Weeren, P.R.; Kaap, J. and Barneveld, A. 2002. Jumping characteristics of naive foals: lead changes and description of temporal and linear parameters. Equine Vet J, 34: 302-307.

Santamaría, S.; Bobbert, M.E.; Back, W.; Barneveld, A. and Van Weeren, P.R. 2004. Evaluation of consistency of jumping technique in horses between the ages of 6 months and 4 years. Am J Vet Res, 65: 945-950. 
Santamaría, S.; Bobbert, M.F.; Back, W.; Barneveld, A. and Van Weeren, P.R. 2006. Can early training of show jumpers bias outcome of selection events? Livest Sci, 102: 163-170.

Schaller, O. 1999. Nomenclatura anatômica veterinária ilustrada. $1^{\circ}$ ed. Ed. Manole. São Paulo. 560 pp.

Schlup, E.; Godoi, F.N.; Andrade, A.M.; Bergmann, J.A.G. e Almeida, F.Q. 2014. Efeito do treinamento sobre a cinemática de equinos no salto de obstáculos. Arq Bras Med Vet Zoo, 66: 1647-1654.
Wallin, L.; Strandberg, E. and Philipsson, J. 2003. Genetic correlations between field test results of Swedish Warmblood Riding Horses as 4 -years-olds and lifetime performance results in dressage and show jumping. Livest Prod Sci, 82: 61-71.

Van Weeren, P.R.; Van de Bogert, A.J. and Barneveld, A. 1992. Correction models for skin displacement in equine kinematic gait analysis. Equine Vet Sci, 12: 178-192. 